\title{
Index of T-wave Variation as a Predictor of Sudden Cardiac Death in Chronic Heart Failure Patients with Atrial Fibrillation
}

\author{
Alba Martín-Yebra ${ }^{1,2}$, Iwona Cygankiewicz ${ }^{3}$, Antoni Bayés-de-Luna ${ }^{4}$, Pablo Laguna ${ }^{2,5}$, \\ Enrico G Caiani ${ }^{1}$, Juan Pablo Martínez ${ }^{2,5}$ \\ ${ }^{1}$ Dipartimento di Elettronica, Informazione e Bioingegneria, Politecnico di Milano, Milan, Italy \\ ${ }^{2}$ BSICoS Group, Aragón Institute of Engineering Research, IIS Aragón, Universidad de Zaragoza, \\ Zaragoza, Spain \\ ${ }^{3}$ Department of Electrocardiology, Medical University of Lodz, Lodz, Poland \\ ${ }^{4}$ Institut Català de Ciències Cardiovasculars, Santa Creu i Sant Pau Hospital, Barcelona, Spain \\ ${ }^{5}$ CIBER en Bioingeniería, Biomateriales y Nanomedicina (CIBER-BBN), Zaragoza, Spain
}

\begin{abstract}
Chronic heart failure (CHF) and atrial fibrillation (AF) are worldwide leading causes of morbidity and mortality in elders, a large part due to sudden cardiac deaths (SCD). The high irregularity of ventricular response in $A F$ patients makes the use of standard SCD-risk markers inappropriate in this target population. The aim of this study was twofold: i) to propose a new index, suitable for AF patients, able to quantify ventricular repolarization changes; and ii) to evaluate its prognostic value in a CHF population with AF. Holter ECG recordings from 176 consecutive $C H F$ patients with $A F$ (22 SCD) were analyzed. The index of $T$-wave variation $\left(I_{\mathrm{TV}}\right)$, quantifying the average $T$-wave changes in pairs of consecutive beats under stable rhythm conditions, was computed using a fully-automatic method. Survival analysis was performed considering $S C D$ as an independent endpoint. $I_{\mathrm{TV}}$ was higher for $S C D$ than non$S C D$ victims (median $(Q 1 ; Q 3)$ : $24.9(14.4 ; 85.4) \mu V$ vs $17.1(11.3 ; 28.2) \mu V, p=0.06)$. In a survival analysis where the threshold was set on the third quartile of $I_{\mathrm{TV}}$ values, $I_{\mathrm{TV}}(+)$ outcome was successfully associated to SCD (Hazard Ratio (CI):3.22 (1.36,7.58) per $\mu V$, $p=0.008)$. In conclusion, we show in this work that $I_{\mathrm{TV}}$ stratifies CHF patients with AF according to their risk of $S C D$, with larger $I_{\mathrm{TV}}$ associated to lower survival probability.
\end{abstract}

\section{Introduction}

Chronic heart failure (CHF) is the leading cause of morbidity and mortality worldwide, representing the major cause of hospitalizations together with atrial fibrillation (AF) in elderly ( $\geq 65$ years) patients. A number of mechanisms supporting both that CHF predispose to AF and that
AF exacerbates CHF are reported [1]. Frequently both disorders coexist, exponentially increasing its incidence and prevalence with age and decreasing quality of life [2].

In particular, the prevalence of AF in patients with mildto-moderate CHF (New York Heart Association, NYHA, classes II and III), ranges from $10 \%$ to $15 \%$ [1]. In this group of patients, a large portion of mortality is represented by sudden cardiac deaths (SCD), most of them being as a consequence of ventricular tachyarrhythmias.

Many questions arise regarding the underlying mechanisms that associate AF, CHF and SCD risk and a better understanding is still needed in order to adopt effective prediction and prevention strategies. Therapeutic options are mainly oriented to an adequate control of ventricular rate and to restore and maintain sinus rhythm. Implantable cardioverter-defibrillators (ICDs), by terminating ventricular tachyarrhythmias, protect from SCD, but identifying patients who benefit the most from this therapy is difficult, especially in the AF subgroup.

The spatio-temporal repolarization heterogeneity of ventricular activity, reflected on the $\mathrm{T}$ wave of the electrocardiogram (ECG), is commonly used to diagnose and assess SCD risk. Ventricular response during AF is highly irregular, mainly influenced by the properties of the atrioventricular (AV) node and atrial electrophysiology. Consequently, this makes not suitable the use of well established non-invasive indices for ventricular repolarization analysis, such as QT dispersion [3], the Tpeak-to-Tend interval [4] or T wave alternans (TWA) [5], as they require certain stability in rhythm to be properly assessed.

Our aim in this study was to propose a new index sensitive to ventricular repolarization changes based on a selective heart rate bin averaging technique as well as to evaluate whether it provides prognostic value in a CHF population with AF. 


\section{Study population}

Consecutive patients with symptomatic CHF corresponding to NYHA classes II and III were enrolled in the multicenter MUSIC (MUerte Súbita en Insuficiencia Cardiaca) study, a prospective study designed to assess risk predictors for cardiovascular mortality in ambulatory CHF patients [6]. The 24-hour Holter ECG recordings of 176 patients (134 males) with AF aged 35-90 years $(68.77 \pm 10.22$ years) were available for the present study. ECG signals were acquired by using SpiderView records (ELA Medical, Sorin Group, Paris, France) and two or three orthogonal leads (X, Y, Z) sampled at $200 \mathrm{~Hz}$ were recorded for each subject. Collection of clinical data for this population was already reported elsewhere in $[6,7]$.

Patients were followed up every 6 months during 48 months. A total of 22 victims of SCD, 24 of other cardiac causes, 20 non-cardiac deaths and 110 survivors were included. SCD, defined as (1) a witnessed death occurring within 60 minutes from the onset of new symptoms unless a cause other than cardiac failure was obvious, (2) an unwitnessed death $(<24$ hours) in the absence of preexisting progressive circulatory failure or other causes of death, or (3) death during attempted resuscitation, was considered as an independent endpoint in this study. Endpoints were reviewed and classified by the MUSIC Study Endpoint Committee. The study protocol was approved by institutional investigator committees and all patients gave written informed consent.

\section{Methods}

We proposed the use of selective beat averaging technique that considers beats preceded by stable RR [8] and computed the average T-wave variation waveform in pairs of consecutive beats defined by bins of stable RR.

\subsection{Preprocessing}

Preprocessing of ECG recordings included heart beat detection and labelling using the Aristotle ECG analysis software [10] and linear filtering of baseline wander. Finally, the ECG was low-pass filtered (with cut-off frequency of $15 \mathrm{~Hz}$ ) to remove noise out of T-wave frequency range and down-sampled. From the 3 orthogonal leads, the vector-magnitud of the VCG signal was computed.

\subsection{Selective beat averaging}

Selective beat averaging was used to obtain averages of P-QRS-T complexes preceded by similar RR intervals $[8,9]$. Based on this, we computed the RR interval series along the 24h Holter recording, the RR interval associated to beat $i$ being defined as the difference between the $i$ and

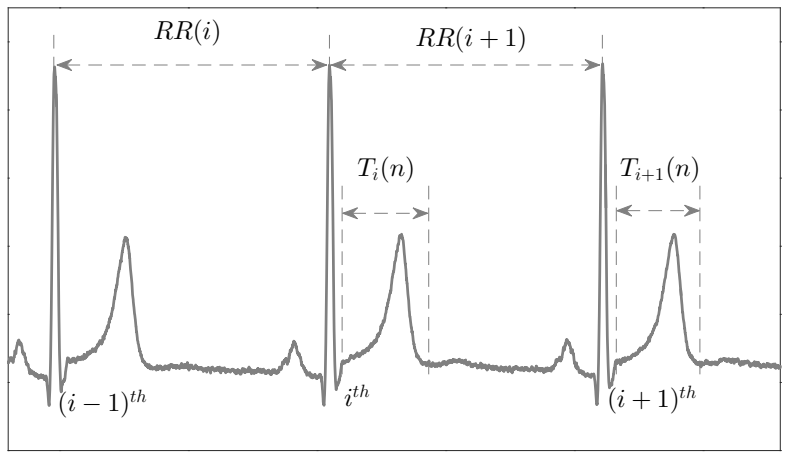

Figure 1. ECG signal with main explicative intervals.

$(i-1) \mathrm{R}$-wave positions, as illustrated on Fig. 1. We then proceeded in this way:

1) Let us define $S$ as the set of all consecutive beat pairs $(i, i+1)$, for any beat $i$ in the Holter recording. $S_{k}$, $k=1, \ldots, K$, are defined as the subsets of beat pairs in $S$ such that $R R(i)$ is within the $k^{t h}$ defined RR interval bin, and the difference between $R R(i+1)$ is close enough to $R R(i)$. That is:

$$
\begin{gathered}
S_{k}=\left\{i:(i, i+1) \in S ; R R(i) \in I_{k},\right. \\
|R R(i+1)-R R(i)| \leq \delta / 2\} \\
I_{k}=\{R R(i): \operatorname{minRR}+(k-1) \delta \leq \\
\leq R R(i) \leq \operatorname{minRR}+k \delta-1\}
\end{gathered}
$$

where $K$ is the total number of bins within the considered $\mathrm{RR}$ range. We used $\delta=40 \mathrm{~ms}$ bins, initially between $\operatorname{minRR}=300 \mathrm{~ms}$ and $\operatorname{maxRR}=1600 \mathrm{~ms}$.

2) At this point, once beats are grouped based on a heartrate criteria and stability can be assumed, the variation waveform associated to the $i^{t h}$ pair of beats in $S_{k}$ was defined as the difference between the two consecutive ST-T complexes. Using vector notation it is:

$$
\Delta T_{k, i}(n)=T_{k, i+1}(n)-T_{k, i}(n)
$$

where vector $T(n)$ denotes the ST-T complex (Fig. 1).

3) In order to make a robust estimation, as this variation of the ventricular activity could be within the range of non-visible microvolt sometimes comparable to the noise level, we replicate the analysis already presented in [11] in the context of TWA analysis, where all TWA waveforms were averaged after being aligned in phase. In this case, the phase-aligned waveform, $\Delta \mathbf{T}_{\mathrm{k}, i}^{a}$, was estimated as:

$$
\boldsymbol{\Delta} \mathbf{T}_{k, i}^{a}=\operatorname{sign}\left(\boldsymbol{\Delta} \mathbf{T}_{k, i}^{\mathrm{T}} \mathbf{w}_{1}\right) \boldsymbol{\Delta} \mathbf{T}_{k, i}
$$

where $\mathbf{w}_{1}$ corresponds to the first eigenvector associated to the greatest eigenvalue $\lambda_{1}$ of the spatial correlation matrix $\mathbf{R}_{\boldsymbol{\Delta} \mathbf{T}_{k}}$ of all $\boldsymbol{\Delta} \mathbf{T}_{k, i}$, (see [11] for more details). 
4) Finally, the T-wave variation waveform associated to the $k^{t h}$ bin, i.e. associated the $S_{k}$ subset, denoted as $\overline{\Delta \mathbf{T}_{k}^{a}}$, is defined as the median waveform of all $\Delta \mathbf{T}_{k, i}^{a}$ computed for that bin.

\subsection{Index of T-wave variation}

The index of T-wave variation $\mathrm{I}_{\mathrm{TV}}$ is then defined as the mean absolute value of the final average waveform of all $\overline{\Delta \mathbf{T}_{k}^{a}}$ :

$$
\mathrm{I}_{\mathrm{TV}}=\frac{1}{N} \sum_{n=1}^{N}\left|\frac{1}{K} \sum_{k=1}^{K} \overline{\Delta \mathbf{T}_{k}^{a}}(n)\right|
$$

with $N$ the total number of samples of the ST-T complex.

\subsection{Statistical analysis}

Data is presented as median $\left(25^{\text {th }} ; 75^{\text {th }}\right.$ percentiles $)$ for continuous variables, unless otherwise specified. MannWhitney test was applied to evaluate differences among groups. Survival analysis was performed by using KaplanMeier estimator and comparison of cumulative events by log-rank test. Prognostic value of $\mathrm{I}_{\mathrm{TV}}$ in predicting SCD was determined with univariate and multivariates Cox proportional hazards analysis. For all tests, the null hypothesis was rejected for $\mathrm{p} \leq 0.05$.

\section{4. $\quad$ Results}

The average distribution of $\mathrm{I}_{\mathrm{TV}}$, now computed for each single RR-bin $S_{\mathrm{k}}$, for both the SCD and non-SCD groups is shown on Fig. 2. One can see that amplitudes differed from one group to the other, especially in the range from 300 to $660 \mathrm{~ms}$, with higher values in the SCD group. From this observation and as a preliminary step, we decided to restrict the $\mathrm{I}_{\mathrm{TV}}$ to this sub-range of RRs for the subsequent analysis (HRs faster than 90 beats/min). The $\mathrm{I}_{\mathrm{TV}}^{300-660}$ was higher for the SCD in comparison to the non-SCD group (24.9 (14.4;85.4) $\mu \mathrm{V}$ vs $17.1(11.3 ; 28.2) \mu \mathrm{V}, \mathrm{p}=0.06)$. It should be noticed here that $\mathrm{I}_{\mathrm{TV}}^{300-660}$ could not be computed in 13 subjects (1 from SCD and 12 from non-SCD group), either because only 2 leads were available ( 8 patients) or the methodology did not select any processable interval (5).

Patients were classified as $\mathrm{I}_{\mathrm{TV}}(+)$ and $\mathrm{I}_{\mathrm{TV}}(-)$, setting the cut point at the third quartile of $\mathrm{I}_{\mathrm{TV}}^{300-660}(32.23 \mu \mathrm{V})$, what successfully predicted SCD (Table 1). In addition to the univariate Cox proportional model, two multivariate models were also constructed by adjusting for significant clinical covariates: (1) age, gender, NYHA class, left ventricular ejection fraction $\leq 35 \%$, and diabetes and (2) use of antiarrhythmic drugs: beta-blockers (103 patients), digoxin (106) and amiodarone (27) plus covariables in 1. For all models, a $\mathrm{I}_{\mathrm{TV}}(+)$ outcome was the only variable associated

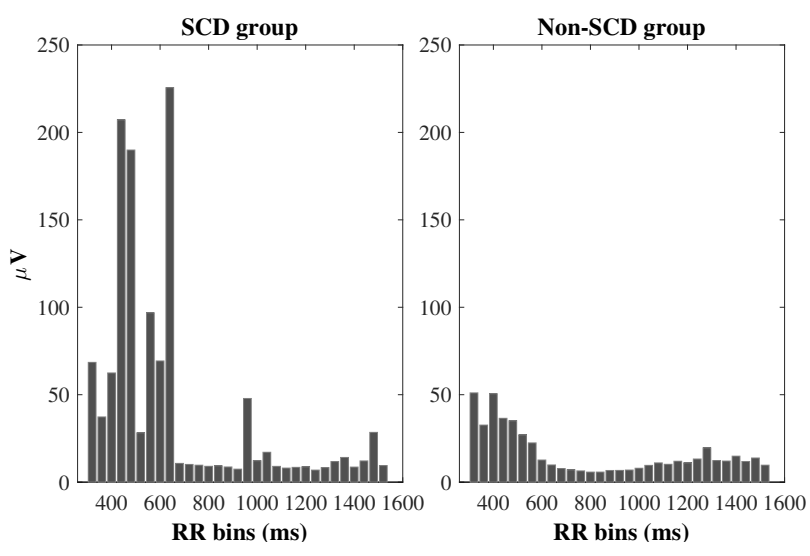

Figure 2. Average distributions of $\mathrm{I}_{\mathrm{TV}}$ for each single RRbin $S_{k}$ in the SCD and non-SCD groups.

with SCD risk, with hazard ratios of 3.22, 3.14 and 3.65 for univariate, multivariate 1 and multivariate 2, respectively. Figure 3 shows the Kaplan-Meier survival curve for SCD.

\section{Discussion and conclusion}

The underlying mechanisms between the association of AF with SCD are intriguing. Indeed, some studies suggested that AF is intrinsically proarrhythmic in the ventricle, increasing susceptibility to ventricular arrhythmias (and, consequently, SCD) whereas others support the hypothesis that AF is actually acting through other risk factors, like $\mathrm{CHF}$, leading to the increase in SCD incidence [12]. A more comprehensive understanding of this phenomena is still needed to better adapt treatment strategies, and to find the answer to in what patients this proarrhythmic risk is higher using noninvasive methods will be of fundamental importance.

In this preliminary study based on observational results, we found that the index of T-wave variation $\mathrm{I}_{\mathrm{TV}}$, quantifying the consecutive T-wave variation based on a selective beat averaging methodology, independently predicted SCD in CHF patients with AF.

In sinus rhythm, the predictive value of several ventricular-based indices, such as QT dispersion [3], the Tpeak-to-Tend interval [4] or TWA [5] among others has been widely demonstrated. However, the high irregularity of ventricular response in AF patients makes the use of those indices not extensive to this pathology. Data on SCD stratification in AF patients based on repolarization indices is still scarce. In a previous study based on HR information including a subset of 155 AF patients from our cohort, authors demonstrated that reduced irregularity of RR intervals in terms of approximate entropy (ApEn) during AF was also predictive of cardiac mortality as opposed to traditional indices [13]. These results encourage to continue research in this field. For example, the evaluation of combined both non-invasive indices of ventricular 
Table 1. Association of $\mathrm{I}_{\mathrm{TV}}^{300-660}$ with SCD in patients with heart failure and atrial fibrillation.

\begin{tabular}{ccccccc} 
& \multicolumn{2}{c}{ Univariate } & \multicolumn{2}{c}{ Multivariate 1* } & \multicolumn{2}{c}{ Multivariate 2** } \\
\cline { 2 - 6 } & HaR $(95 \% \mathrm{CI})$ & p-value & HaR $(95 \% \mathrm{CI})$ & $\mathrm{p}$-value & HaR $(95 \% \mathrm{CI})$ & $\mathrm{p}$-value \\
\hline $\mathrm{I}_{\mathrm{TV}}^{300-660} \geq 32.23$ & $3.217(1.36,7.58)$ & $\mathbf{0 . 0 0 8}$ & $3.148(1.32,7.49)$ & $\mathbf{0 . 0 1}$ & $3.652(1.46,9.11)$ & $\mathbf{0 . 0 0 5}$ \\
\hline
\end{tabular}

* Adjusted model includes age, gender, New York Heart Association class, left ventricular ejection fraction $\leq 35 \%$, and diabetes.

** Multivariate 1 plus the use of antiarrhythmic drugs: beta-blockers ( $\mathrm{n}=103)$, digoxin (106) and amiodarone (27)

dispersion together with HR-derived indices, may improve this prediction. Also previous observations that short-longshort beat sequences may lead to VT/VF episodes [14] suggest the possibility of evaluating ventricular repolarization activity by extending the methodology to other beat sequences. Improving the performance of SCD-risk stratification by using non-invasive methods is one of the main problems that public health systems are facing nowadays.

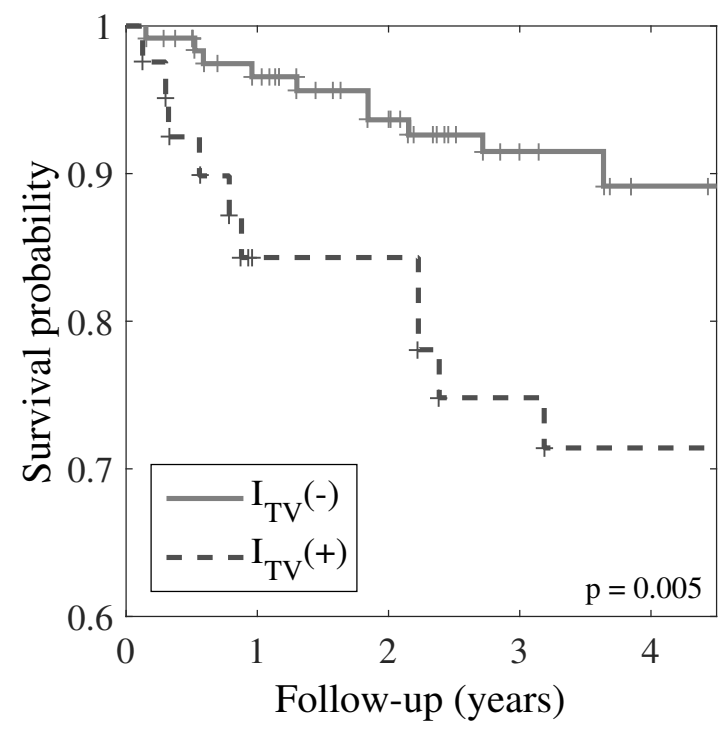

Figure 3. Long-term survival curves for sudden cardiac death according to $\mathrm{I}_{\mathrm{TV}}(+)$ and $\mathrm{I}_{\mathrm{TV}}(-)$ outcomes.

\section{Acknowledgements}

This work was supported by CIBER in Bioengineering, Biomaterials \& Nanomedicne (CIBER-BBN) through Instituto de Salud Carlos III and FEDER (Spain), project TEC2013-42140-R funded by MINECO and FEDER, and by Gobierno de Aragón and European Social Fund (EU) through BSICoS Group (T96). The computation was performed by the ICTS NANBIOSIS, specifically by the High Performance Computing Unit of the CIBER-BBN at the University of Zaragoza. Partial support also from the Italian Space Agency (contract 2013.33.R.0, recipient E.G.Caiani).

\section{References}

[1] Van Den Berg MP, Tuinenburg AE, Crijns HJGM et al. Heart failure and atrial fibrillation: current concepts and controversies. Heart 1997;77:309-313.

[2] Maisel WH, Stevenson LW. Atrial Fibrillation in Heart Failure: Epidemiology, Pathophysiology and Rationale for Therapy. Am J Cardiol 2003;91:2D-8D.

[3] Day CP, McComb JM, Campbell RW. QT dispersion: an indication of arrhythmia risk in patients with long QT intervals. Br Heart J 1990;64:342-44.

[4] Couderc JP, Zhou M, Sarapa N, Zareba W. Investigating the effect of sotalol on the repolarization intervals in healthy young individuals. J Electrocardiol 2008;41(6):595-602.

[5] Verrier RL et al. Microvolt T-wave alternans physiological basis, methods of measurement, and clinical utility - consensus guideline by International Society for Holter and Noninvasive Electrocardiology. J Am Coll Cardiol 2011;58(13):1309-24.

[6] Vázquez R, Bayés-Genís A, Cygankiewicz I, et al. The MUSIC risk score: a simple method for predictiong mortality in ambulatory patients with chronic heart failure. Eur Heart J 2009;30:1088-1096.

[7] Cygankiewicz I, Zareba W, Vázquez R, et al. Risk stratification of mortality in patients with heart failure and left ventricular ejection fraction $>35 \%$. Am J Cardiol 2009;103:1003-1010.

[8] Badilini F, Maison-Blanche P, Childers R, Coumel P. QT interval analysis on ambulatory electrocardiogram recordings: a selective beat averaging approach. Med Biol Eng Comput 1999;37:71-9.

[9] Caiani E G, Pellegrini A, Bolea J, et al. Impaired T-wave amplitude adaptation to heart-rate induced by cardiac deconditioning after 5days of head-down bed-rest. Acta Astronaut 2013;91:166-72

[10] Moody GB, Mark RG. Development and evaluation of a 2-lead ECG analysis program. Computers in Cardiology 1982;9:39-44.

[11] Martín-Yebra A, Caiani EG, Laguna P, Monasterio V, Martínez JP. Circadian Modulation on T-wave Alternans Activity in Chronic Heart Failure Patients. XLII International Conference on Computing in Cardiology 2015;42:845-848.

[12] Reinier K, Marijon E, Uy-Evanado A et al. The association between atrial fibrillation and sudden cardiac death: The relevance of heart failure. JACC Heart Fail 2014;2:221-227.

[13] Cygankiewicz I, Corino V, Vázquez R, et al. Reduced Irregularity of Ventricular Response During Atrial Fibrillation and LongTerm Outcome in Patients with Heart Failure. Am J Cardiol 2015;116:1071-1075

[14] Denker S, Lehmann M, Mahmud R et al. Facilitation of ventricular tachycardia induction with abrupt changes in ventricular cycle length. Am J Cardiol 1984;53:508-515.

Address for correspondence:

Alba Martín

DEIB, Politecnico di Milano Piazza Leonardo da Vinci, 32, 20.133, Milano, Italy

albapilar.martin@polimi.it 